\title{
Study on Cowboy Process Printing Technology and Effects Xiaorong Deng
}

\author{
Jiangxi Institute of Clothing Technology, Fashion Design Branch, Nanchang \\ 2930731380@qq.com
}

Keywords: Printing denim; Printing process; Design; Traditional crafts; Fashion

\begin{abstract}
Cowboy culture has a long history because of its inclusive features make the heritage of 150 years, so it is loved by more and more people. Today, with the continuous progress of the times, the traditional cowboy printing has been unable to meet the modern clothing aesthetic requirements. Jeans need innovation in order to keep brilliant. Based on analysis of several aspects of cowboy printing process, such as hand-painted, rubbing, discharge printing, handicraft flowers, overprint coating, transfer printing, flocking, burnt-out printing, digital printing, laser engraving and so on, this paper studies advantages and disadvantages of each aspects by way of examples as well as their respective scope. Final conclusion: jeans must select an appropriate printing process according to fabrics performance combined with new modern printing technology. Only the combination of traditional and modern technology, can jeans get new blood and culture and develop better.
\end{abstract}

\section{Introduction}

Blue-collar favorite jeans, because production of denim fabric in the 19th century gives it tough wear, thick rough cloth body, stiffness characteristics. In the modern cowboy make improvements, be it fashion design to make it more fashionable, changes to diversify the structure of the organization, a diversified processing composition, the effect of functional fabrics series, art garment washing process in these aspects of technology innovation. After innovation jeans has become a popular favorite global apparel product. It increasingly meets people seeking personalized, fashion, and nostalgic, natural aesthetic needs.

Heritage of centuries of jeans has been at the front of fashion, denim is the beginning of a unique wearing gold worker, in the form of canvas overalls appears. Over the years the only indigo denim fabric from now a wide variety of colorful fabric, the fabric is also turned rough from wear elastic, thin knit quality. Clothing continuous innovation. Denim appeal continues, because it has the resilience to adapt to the aesthetic of the times. Printing apparel has been followed in those years, while fashion designers have begun to set off a pioneering new jeans look atmosphere. On d'NIM 2016 spring and summer denim conference, Chinese designer Chen Wen using a large area of water, printing and stitching, denim fabrics and painting the match turned into a work of art depicting the flow of oil. Printing a variety of craft techniques, combining it with the cowboy to make it more personal, more wild, with Balmain, Chanel, VivienneWestwood these brands, for example, they are often put into the printing denim couture.

\section{Traditional Printing Process}

Printing jeans first appeared in the train conductor stripes printed denim uniforms, in the 1940s. Later, on the use of sports and leisure clothing colorful Aloha "Hawaiian pattern is Levi's creative 50s 70s became popular hand-painted jeans, cowboy stamp has been changing to adapt to modern trends

Hand Printing. Letterpress printing and stencil printing is hand printing two common ways. Template engraving pattern is the use of letterpress printing mode, the pattern formed on the denim fabric is the use of its yin and yang in the above rubbing. Also known as the stencil printing stencil printing, using filigree patterns on different materials, in the hollow places Scraping color fabrics. Hand printing with respect to industrial printing, the low efficiency, low yield, and uneven quality showed significant deficiencies. But for now the promotion of industrial production mode, hand 
printing performance advantages of its unique, creative and artistic value.

Hand Painted. The performance of individual young people today can be expressed by way of hand-painted, using a pigment or dye to paint on jeans. Depending on the hand-painted brush tool use, the use of brush strokes on a denim dress expression sketched, painted flat, spill effect of making jeans unique. In order to avoid damage to finishing the pattern of colorful, hand-painted cowboy-style clothing color usually directly after washing.

Discharge Printing. After the surface has been dyed denim fabric added carved white powder or other agent to break them dial background is discharge printing applications. White blue effect formed by screen printing on a printing paste containing Stubbs color, the use of drying, steaming, washing, etc. Process made, jersey T-shirt and jeans soft and elastic denim fabric more like application of discharge printing, like most classic application of traditional floral pattern and geometry, because it is difficult to control the printing process, if the improper operation of the process, the original surface easily penetrate clothing color, colorful overprint coatings to a lesser extent, so more for the production of traditional flower pattern.

Overprint Coatings. Utilization in denim fabric pattern printed on Rotary's Peace Network, application formulated pigment paste is overprint coatings. It has the advantage of good coverage, use less fabric in denim jacket friction flat portion in the back printing more. It is light in texture, soft and fluffy fabric does not apply. The white inorganic pigment and a specific Bo Heli formulated as overprint white pulp, and then followed by the application of other pigments or additives dubbed printing paste. Get bright colors on a dyed fabric printing methods; the use of white pigment-free seven can improve pigment printing opacity. White iron and aluminum are two elements white inorganic pigment commonly used iron white powder for white overprint; aluminum color printing is applicable, when it is mixed with other pigments to fight hair color and good performance. The advantage is no waste water, saving chemicals, the process is simple. It is made out of effect and anti-pollution, discharge printing products similar. It is used in more punk and pop pattern。

Transfer Printing. Transfer printing sublimation transfer printing, the melting method transfer printing, transfer printing method of stripping, semi-wet transfer printing four kinds of processes, it refers to the reuse on special paper substrate with disperse printed pattern printed pattern on hot denim fabric is the use of a method which does not require additional processing before and after the operation is simple, a high degree of color reduction, but also more environmentally friendly printing methods, the use of transfer manner, is widely used in the market currently.

Flocking. Printed target pattern on the denim fabric and electrostatic flocking after the adhesive paste, the deal is finally flocking printing process. In the high-end denim clothing flocking printing more popular application, multi-application European retro flowers and vines. It enables the fabric to produce a strong sense of three-dimensional convex, feel soft pattern strong sense of hierarchy ladder, but it produces a small amount of product, flocking inappropriate scrub through force, when designs as simple as possible, not suitable too fine.

Foam Printing. Three-dimensional printing is another name called foam printing, the printing process on the basis of glue extending from the foam printing, it is certain proportion of several chemicals high coefficient of expansion, drying in the printing mortar printing dye parts of the 200-300 degree heat blistering, so that patterns showing a three-dimensional effect, similar relief. Printing paste on the fabric using a thermoplastic resin, a foaming agent, three-dimensional relief effect by high temperature steam foaming process. Foam printing finished effect is very good, it is widely used in cotton, denim, expanded its printing surface projecting three-dimensional sense. But it is difficult to handle at the edge of the fabric pattern on cartoon letter pattern which is widely used. (Fig. 2) 


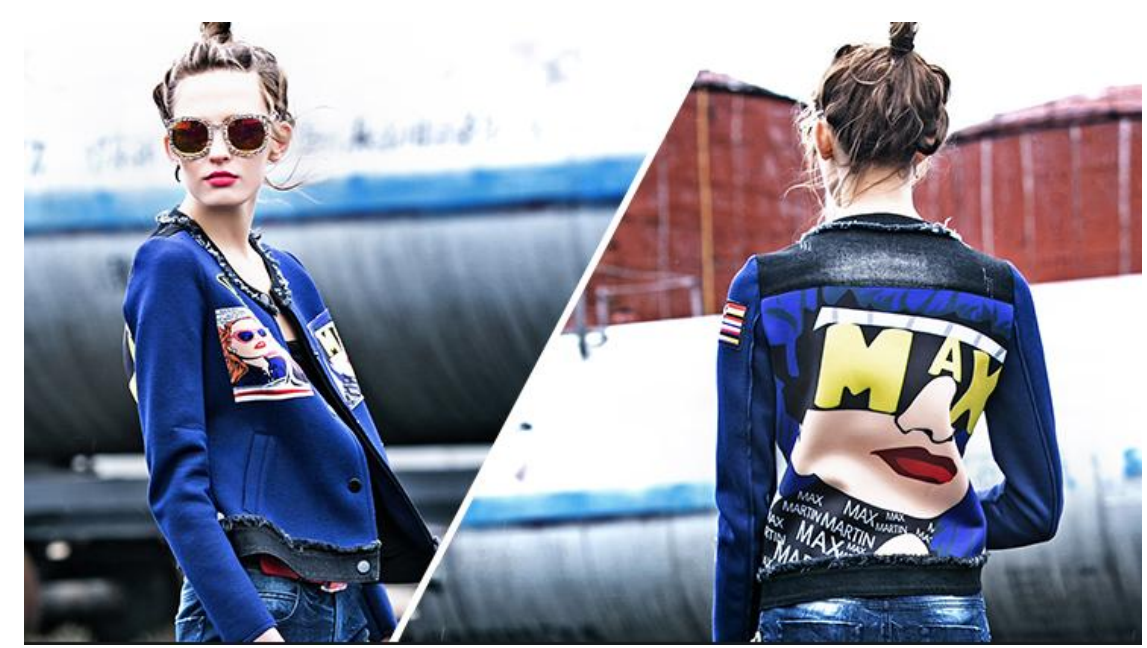

Figure 2. Mama ti new European style fashion denim jacket printing stitching female street Tide brand casual jacket

\section{Modern Printing Technology}

Modern printing technology and computer complementary, no plate, setting commands on the computer to generate patterns.

Digital Printing. A realistic printing pattern on digital printing denim fabric is formed by computer processing. With the traditional flat, rotary screen printing very different. Personalization and customization is the development trend of modern cowboy clothing. Application of digital printing technology is digital printing. Digital printing is a high-tech birth was that modern society with the development of computer technology continues to gradually develop the collection machinery, computer technology integration. Parallel to this emerging technology is constantly improving, its emergence is an innovation in itself, its development and to the textile industry infused new blood brought a new development.

Digital printing in small quantities, multi-species mode of production to meet changing market demands and pop elements, so that the pace of keeping up with fashion cowboy stamp. It can be customized personalized products and industrial production numbers, as opposed to the traditional printing to reduce the pollution of the environment, layered pattern, rich pattern, no plate. But its drawback is deep indigo denim background, difficult to control the color, the higher production costs. d'nim latest brand new autumn and winter denim jeans recently released in Beijing, the brand is using the latest digital printing technology, designers contrasting color printing, scattered segmentation and decent stitching, it is the three-dimensional geometric prints and modernization color combination show in front of everyone.

Laser Engraving. Production of computer instructions laser carving, denim fabric pattern formed on the surface by laser ablation of the dye formed by the decomposition gasification. Compared with traditional laser printing process reduces pollution potassium permanganate spray process is relatively simple, produce whiskers, monkeys, torn, distressed, washed widely applied decorative effect. It features accurate copy in the fabric, and finished a clear pattern. It is relative to the traditional printing process to form a broader pattern style, three-dimensional effect is stronger. Due to the limitations of the works, the effect of laser engraving process can only be formed depth background.

\section{Cowboy Printing Trends}

Cowboy ever-changing style, style is also varied, naturally everyone's favorite cowboy, retro, nostalgic features, it does not require functional. The future development trend of cowboy grafted his textile 91, the concept of finishing method, into high technology, adding some special additives, the fusion of art washing clothing manufacture it without losing the original style trend. 
Today denim fabric rich visual effects through a variety of printing processes and patterns to the rich. It is in line with the performance needs of the public, at this stage of the advantages of low cost, high efficiency. Printed rich denim colors, ornate rococo, retro flower vine European Man, paisley patterns, still fresh and natural Hawaiian Aha rib-like, abstract geometric patterns, these patterns blend relationship denim and flowers. They complement each other forming a new feature.

\section{Conclusion}

This article analyzes the various types of cowboy printing methods, advantages and disadvantages of the production mode, and textured style pattern. Then it summarizes several commonly used cowboys printing process. Cowboy is becoming a stylized art, which meets personalized clothing demands. The combination of traditional techniques and modern technology, has important influences on cowboy cultural heritage and future development.

\section{Acknowledgements}

2014 Jiangxi Social Sciences project "based on the clothing of Jiangxi Regional Culture Regional Industry and Research" initial results, project number: 14YS21

\section{References}

[1] lu Bao. Digital printing and traditional printing [J]. Screen printing, 2011 (3): 20-24.

[2] Yifei media. Cowboys [M]. Nanjing: Jiangsu Fine Arts Publishing House, 2005.

[3] PeiChen Qing. Modern clothing denim fabric design application [J]. Textile Research, 2011, 32 (7): 117-121.

[4] Gao pan. Bo Gao. Li Zhongyuan. Printing design [M]. Wuhan: Hubei Fine Arts Publishing House, 2006.

[5] Guofeng Li. Washed denim garments trial technology [M]. Beijing: China Textile Press, 2014.

[6] Fujun Wan. Washed denim garments trial technology [M]. Beijing: China Textile Press, 2014.

[7] Qingyun Jiang. When the denim fabric meets cold transfer printing technology [J]; Textile and Apparel Weekly; 201233

[8] Zhongqi Zhang. Second design jeans [J]; International Textile Leader; 201003

[9] Hanzhou Wang. Impact printing process of printing graphic design [J]; dyeing and finishing technology; 201002

[10]Keli Wang; Jiang Qingyun. Denim fabric industry status and development of new trends [J]; Textile Leader; 201010 